\title{
Open Source Web Software Architectures for Geographic Information Systems: A Systematic Mapping Study
}

\section{Alvaro Uyaguari, Edison Espinosa-Gallardo, Milton Escobar-Sánchez, José Luis Carrillo-Medina, and Patricio Espinel}

Universidad de las Fuerzas Armadas ESPE, Av. General Rumiñahui s/n, 171-5-231B Sangolquí, Ecuador

\section{Abstract}

A geographic information system is an organized integration of hardware, software and geographic data designed to collect, store, manipulate, analyze and present geographically referenced information. In the development of applications web the software architecture are very important aspect when developing complex software

Corresponding Author: Alvaro Uyaguari aduyaguari@espe.edu.ec

Received: 28 July 2017 Accepted: 5 September 2017 Published: 30 January 2018

Publishing services provided by Knowledge E

(c) Alvaro Uyaguari et al. This article is distributed under the terms of the Creative Commons

Attribution License, which

permits unrestricted use and redistribution provided that the original author and source are credited.

Selection and Peer-review under the responsibility of the SIIPRIN Conference Committee.

\section{G OPEN ACCESS} efficiently. This research shows a mapping study which objective is know the state of the art of the software architectures of the geographic information systems for open source web environments. The results show that $74.19 \%$ of contributions are integrations of existing open source components, $16.13 \%$ add tools to the software architecture, and $9.68 \%$ integrate new methods to solve or improve algorithms that are part of the architecture. This indicates that further research is needed to experience new tools and/or new algorithms to solve or improve existing ones.

Keywords: Web software architectures, open source, geographic information systems, systematic mapping study.

\section{Introducción}

La información geoespacial es resultado del procesamiento de los datos de objetos y hechos con referencia espacial. Actualmente, es una componente esencial de la infraestructura nacional e internacional para la sociedad de la información. Se estima que alrededor del 80 por ciento de nuestras decisiones diarias dependen de esta [1], que es procesada por los sistemas de información geográfica (SIG). Estos están formados por un conjunto de herramientas que integra y relaciona diversos componentes (usuarios,?hardware,?software y procesos)[2], que permiten la adquisición, el almacenamiento, la manipulación, el análisis, la visualización y la difusión de información geoespacial [2]. Facilitando la incorporación de aspectos sociales-culturales, 
económicos y ambientales, que conducen a la toma de decisiones de una manera más eficaz [2].

Los SIG se caracterizaron por ser sistemas cerrados, monolíticos y propietarios mientras que hoy integran varios componentes que permiten disponer de aplicaciones versátiles que trabajan de forma transparente. Estos utilizan la World Wide Web (WWW o la web) como el medio de conectividad principal en la computación distribuida en TI en general y en el dominio del geo procesamiento específicamente [3, 4]. Estos se centraron en los datos y herramientas implementadas en una arquitectura clienteservidor, evolucionando a un modelo de servicio web [5], donde la arquitectura web no solamente se centra en entregar los datos sino también la funcionalidad de geo procesamiento (componentes). Lo cual permitió satisfacer la ubicuidad, la facilidad de acceso, la flexibilidad y la reducción de riesgo de aislamiento y obsolescencia. Creando servicios y/o aplicaciones más amplias y completas [6]. Extendiéndose los beneficios de los SIG ampliamente en la web [7].

El desarrollo de una arquitectura de software para un SIG se basa en especificaciones que permiten normar la gestión y el manejo de la información geográfica [8]. Por ejemplo, el Open Geoespacial Consortium (OGC) brinda especificaciones para la aviación, construcción de infraestructuras, modelamiento 3D, inteligencia del negocio, defensa militar, gestión de respuesta a emergencias y desastres, energía, geociencias y medioambiente, infraestructura de datos espaciales y gobernabilidad, Internet Móvil y servicios de localización, sensores web, investigación [9].

El objetivo de esta investigación es realizar un estudio de mapeo sistemático (EMS) (EMS es un método de investigación basado en la evidencia, que proporciona una visión general de un área de investigación, e identifica la cantidad, el tipo de investigación y los resultados disponibles dentro de ella [10]), sobre la arquitectura de software web de código abierto para sistemas de información geográficos, que permitió del análisis de un conjunto de artículos, establecer la problemática y aportes sobre la investigación planteada.

El artículo está estructurado de la siguiente manera: En la sección 2, se describe la pregunta de investigación y la metodología propuesta. En la sección 3, se detalla la planificación del mapeo. En la sección 4, se realiza la ejecución del mapeo sistemático. El análisis y discusión de resultados son detallados en la sección 5. Las situaciones que amenazan la validez de esta investigación son presentadas en la sección 6 . Para finalizar, en la sección 7 se establecen las conclusiones de la investigación. 


\section{Metodología de la Investigación}

Dirigimos el alcance de este mapeo hacia las tecnologías web de los sistemas de información geográficos desde el año 2012 hasta el mes de junio de 2017. La pregunta de investigación que se planteo es la siguiente:

R1Q1: ¿Qué tipos de investigación y contribuciones que se han realizado en los últimos 5 años en la arquitectura de software Web Gis Open Source?

La R1Q1 busca mapear la distribución de los tipos de investigación y contribución llevadas a cabo en los últimos 5 años en arquitecturas de soluciones informáticas Web Open Source que gestionen información geográfica. Se realiza un MSL (Systematic Mapping Study, SMS en inglés) cubriendo las actividades de planificación y ejecución para identificar la cantidad y tipo de publicaciones disponibles sobre esta temática. Según [11], los pasos para llevar a cabo un mapeo sistemático son: la definición de la pregunta de investigación, la búsqueda de artículos relacionados, el análisis de resultados, la selección de artículos, el keywording del resumen y la extracción y mapeo de los datos.

En cada uno de estos pasos se producen ciertos resultados que finaliza con el mapa de los artículos relacionados sobre la temática planteada. Las búsquedas de artículos incluyen un conjunto de reglas, actividades, elementos e instrumentos, que formalizan y dan soporte a las actividades de:

- Planificación del Mapeo Sistemático.

- Selección de fuentes de artículos.

- Definición de la cadena de búsqueda.

- Definición de los criterios de inclusión y exclusión.

• Ejecución del Mapeo Sistemático.

- Preselección de artículos.

- Selección de artículos.

- Extracción de datos de los artículos.

La selección de fuentes de artículos requiere un conjunto de reglas para garantizar la calidad y cantidad de los artículos. Las reglas son: definición de criterios de selección de fuentes y motores de búsqueda. En la definición de cadenas de búsqueda, estructuraran las cadenas de búsqueda utilizadas para responder a la pregunta de investigación. Como lo propone [12], la cadena de búsqueda se construye a partir 
de la pregunta de investigación planteada con la estrategia PICOC y para refinar la pregunta de investigación se aplica las estrategias propuestas por [13]. La definición de los criterios de inclusión y exclusión son establecidos previamente de acuerdo a un conjunto de criterios utilizados durante la actividad de ejecución de la búsqueda para la preselección y selección de artículos. En la preselección de artículos se procede a la lectura de algunas secciones del artículo, a fin de identificar en el texto términos utilizados en la cadena de búsqueda. En la selección de artículos se realiza una lectura completa del artículo, aplicándose los términos de la cadena de búsqueda. Finalmente, para la extracción de datos de los artículos, se crea un instrumento que permite recolectar la información relacionada con la temática.

\section{Planificación del Mapeo Sistemático}

La planificación constituye el soporte a las actividades de organizar, ejecutar y entregar resultados de la búsqueda de artículos. A continuación, se describen las actividades, criterios instrumentos entre otros para llevar a cabo esta actividad.

Рara la selección de fuentes de artículos se implantó las siguientes reglas:

(a) Criterios de selección de las fuentes:

- Artículos originados en fuentes fiables como revistas, revistas científicas, conferencias internacionales y capítulos de libros.

(b) Idioma:

- Artículos escritos en el idioma inglés.

(c) Motores de Búsqueda:

\subsection{Artículos buscados en Scopus y IEEE Xplore.}

En la actividad definición de la cadena de búsqueda se establece la cadena que se usa para responder la pregunta de investigación, fundamentándose en la estrategia PICOC: Population, Intervention, Comparison, Output, Contexto [13]; quedando la pregunta de investigación planteada de la siguiente forma:

- Población: Sistemas de información geográficos.

- Intervención: Arquitectura del software.

- Contexto1: Software abierto. 
- Contexto2: Web

- Comparación: Software open source usados en la arquitectura.

- Salida: Estadística de componentes de software usados en la arquitectura web de

de los Sistemas de Información Geográficos.

Definidos los términos según PICOC, población, intervención y el contexto, se procedió a definir la estructura de la cadena. Utilizamos el término geographic information systems web Open Source para la población y sus correspondientes sinónimos de acuerdo con [14]. Рara la intervención se utilizó software architecture [15]. Рara el contexto1 se utilizó el término open source, mientras que para el contexto 2 se empleó "Web Gis" debido a que en un muestreo aleatorio, todos los artículos relacionados con la publicación de información geográfica en la web incluían la palabra clave "webgis o su sinónimo web-gis". Mas no se incluyó "Program architecture" debido a que no afectó en ningún aspecto a incorporar o excluir investigaciones.

En la Tabla 1 se detallan los sinónimos relacionados con los términos de la pregunta de investigación.

TABLA 1: Sinónimos y términos relacionados de búsqueda de artículos PICOC.

PICOC
Población.
Intervención.
Contexto 1.
Contexto 2.

Término
Geographic information
systems.
Software Architecture.
Open Source.
Webgis.

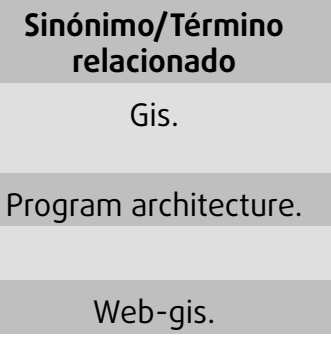

Al momento de establecer la cadena se establecieron cuatro subcadenas de búsqueda, utilizando operadores lógicos entre los componentes de PICOC para relacionar los términos y sus sinónimos correspondientes.

La Tabla 2 muestra las actividades y los insumos de la planificación para la ejecución de la búsqueda de los artículos relacionados con el objeto de estudio.

De la pregunta de investigación planteada se determinan los criterios de inclusión y exclusión, dichos criterios se utilizan en la ejecución de la búsqueda de preselección y selección de los artículos.

En la actividad de preselección de artículos, los criterios de inclusión permiten incorporar artículos en los que se evidencie el uso de términos de la cadena de búsqueda en las secciones como: título del trabajo, resumen y palabras claves. Por lo contrario los criterios de exclusión permiten descartar los artículos en los que se utilizan los 
TABLA 2: Actividades e insumos de planificación.

\begin{tabular}{|c|c|c|}
\hline No. & Actividad & Insumos de Planificación \\
\hline 1 & $\begin{array}{l}\text { Seleccionar Fuentes } \\
\text { para la Búsqueda de } \\
\text { Artículos }\end{array}$ & $\begin{array}{l}\text { Fuentes: Revistas, capítulos de libros, artículos de revistas. } \\
\text { Idioma: Inglés. Motores de Búsqueda: Scopus y IEEE } \\
\text { Xplore. }\end{array}$ \\
\hline 2 & $\begin{array}{l}\text { Definición de los } \\
\text { criterios de inclusión y } \\
\text { exclusión. }\end{array}$ & $\begin{array}{l}\text { Qué tipos de investigación y contribuciones que se han } \\
\text { realizado en los últimos } 5 \text { años en la arquitectura de } \\
\text { software Web Gis Open Source? }\end{array}$ \\
\hline 3 & $\begin{array}{l}\text { Preselección de } \\
\text { artículos. }\end{array}$ & $\begin{array}{l}\text { Criterios de inclusión: Evidencia del uso de los términos de } \\
\text { la cadena de búsqueda en el contenido del artículo. Criterio } \\
\text { de exclusión: No se evidencia el uso de los términos de la } \\
\text { cadena de búsqueda en el contenido del artículo }\end{array}$ \\
\hline 4 & Selección de artículos. & $\begin{array}{l}\text { Criterios de inclusión: Evidencia de la aplicación de } \\
\text { Sistemas de Información Geográficos Web Open Source. } \\
\text { Criterios de exclusión: No se evidencia de la aplicación de } \\
\text { Sistemas de Información Geográficos Web Open Source. }\end{array}$ \\
\hline 5 & $\begin{array}{l}\text { Definición de la } \\
\text { cadena de búsqueda. }\end{array}$ & $\begin{array}{l}\text { Cadena_Resultamte = "geographic Information systems" } \\
\text { or "gis") AND "software architecture" AND "open source" } \\
\text { AND "web-gis" OR "webgis"). }\end{array}$ \\
\hline 6 & Extracción de datos & Información básica de los artículos seleccionados. \\
\hline
\end{tabular}

términos de la cadena de búsqueda, pero que no corresponden al contexto de Sistemas de Información Geográficos Web Open Source. Esta actividad genera una lista que contiene los estudios primarios de la búsqueda.

En la actividad de selección de artículos se toman los criterios de inclusión para incorporar los artículos en los que se evidencie actividades sobre la temática de Sistemas de Información Geográficos Web Open Source. En cambio, se aplican los criterios de exclusión para descartar los artículos en los que no se detallen Sistemas de Información Geográficos Web Open Source, para ello se realiza una lectura completa de los artículos.

\section{Ejecución del Mapeo Sistemático}

En esta actividad se lleva a cabo las tareas planificadas, para buscar artículos relacionados con la temática planteada, se inicia ingresando la cadena (Cadena_Resultante) en las bases digitales IEEE y Scopus. La Tabla 3 muestra los resultados de la ejecución de la Cadena_Resultante, filtrándose los resultados a través del título, abstract y keywords correspondientes, obteniéndose 25 artículos en Scopus y 17 artículos en IEEE Xplore. Se aplican los criterios de inclusión y exclusión determinados para la selección de artículos, obteniéndose 19 artículos de Scopus y 12 de IEEE Xplore. Cabe destacar que la columna precisión en la Tabla 3, es la relación porcentual entre el número de artículos Seleccionados versus el número de artículos localizados. 
TABLA 3: Artículos localizados, seleccionados y precisión en relación a la cadena de búsqueda Cadena Resultante.

\begin{tabular}{|c|c|c|c|}
\hline Motor & $\begin{array}{c}\text { Artículos } \\
\text { Localizados }\end{array}$ & Artículos Seleccionados & Precisión \\
\hline Scopus & 25 & 19 & $61,29 \%$ \\
\hline IEEEXplore & 17 & 12 & $38,71 \%$ \\
\hline Total & 42 & 31 & \\
\hline
\end{tabular}

Luego de realizar un análisis previo, se excluyeron aquellos artículos que no evidencian en su contenido la aplicación de Arquitecturas de Software Open Source para Sistemas de Información Geográficos. Los artículos seleccionados se detallan en la sección referencias de la publicación.

La revisión de los 31 estudios primarios permitió clasificarlos de acuerdo a los tipos de investigaciones, que se muestra en la Tabla 4. Mientras, en la Tabla 5 se muestra las contribuciones de las investigaciones para establecer la arquitectura de software de los sistemas de información geográficos con herramientas open source.

TABLA 4: Tipos de investigación.

\begin{tabular}{l|c|c|c|}
$\begin{array}{l}\text { Tipo de } \\
\text { investigación }\end{array}$ & Casos & Porcentaje & Referencias \\
\hline Case Study & 27 & $87,10 \%$ & $\begin{array}{c}{[1],[3],[4],[5],[6],[7],[8],[9],} \\
{[10],[12],[13],[15],[17],[18],,[19],} \\
{[20],[21],[22],[23],[24],[25],[26],} \\
{[27],[28],[29],[30]}\end{array}$ \\
& & & $\begin{array}{c}{[26} \\
\text { Experiment }\end{array}$ \\
\hline Action Research & 3 & $9,68 \%$ & {$[14],[16]$}
\end{tabular}

A partir de la información de la Tabla 4, se calculó las frecuencias de las publicaciones en cada tipo de investigación. Se utilizó un diagrama de burbujas para representar las frecuencias interconectadas, como se muestra en la Fig. 1. Esto es básicamente un diagrama de dispersión $x-y$ con las burbujas en las intersecciones de las categorías.

TABLA 5: Contribuciones de las investigaciones.

\begin{tabular}{l|c|c|c}
$\begin{array}{l}\text { Contribución } \\
\text { Herramienta }\end{array}$ & Número & Porcentaje & Referencias \\
$\begin{array}{l}\text { Herramienta y } \\
\text { método }\end{array}$ & 5 & $16,13 \%$ & {$[6],[13],[14],[15],[17]$} \\
& 23 & $74,19 \%$ & {$[1],[2],[3],[4],[5],[8],[9],[10]$,} \\
& & & {$[12],[16],[19],[20],[21],[22],[23]$,} \\
{$[24],[25],[26],[27],[28],[29],[30]$,} \\
Método
\end{tabular}




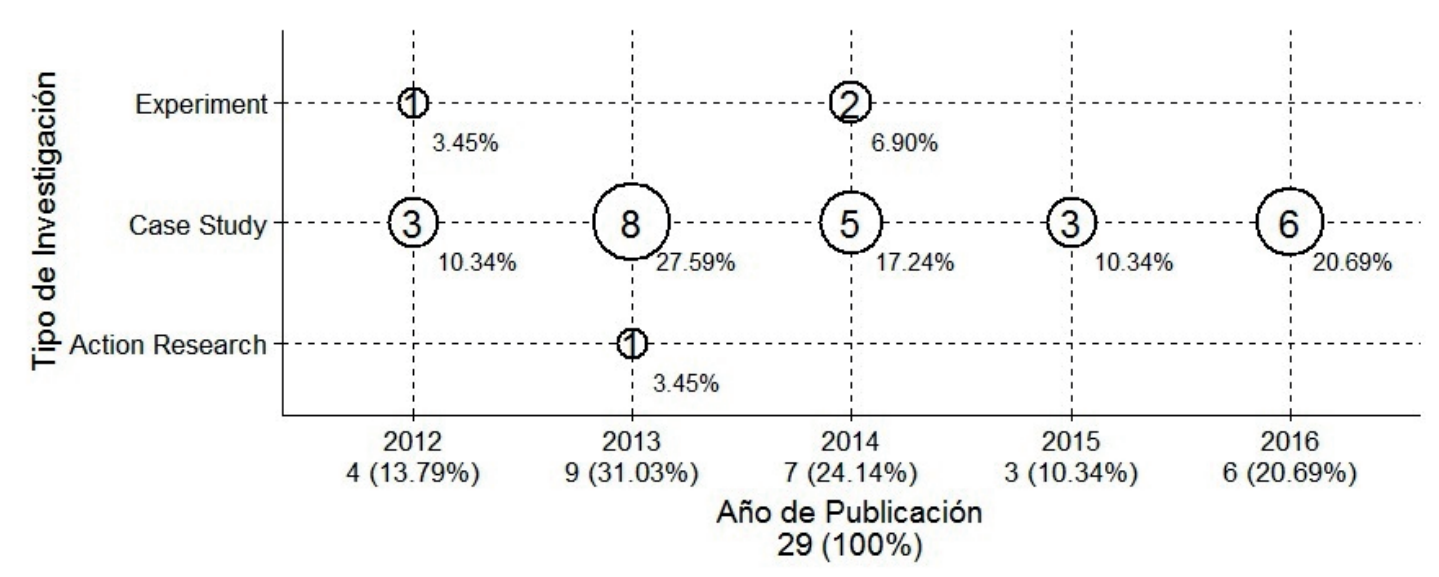

Figura 1: Mapa de los años de publicación de acuerdo a los tipos de investigación en la arquitectura de software Web Gis Open Source.

El la Fig. 2 se evidencia que la mayor parte de contribuciones en el periodo de estudio, son herramientas y métodos de implementación de arquitecturas en base a la reutilización de componentes open source y siguiendo un método establecido en el estudio.

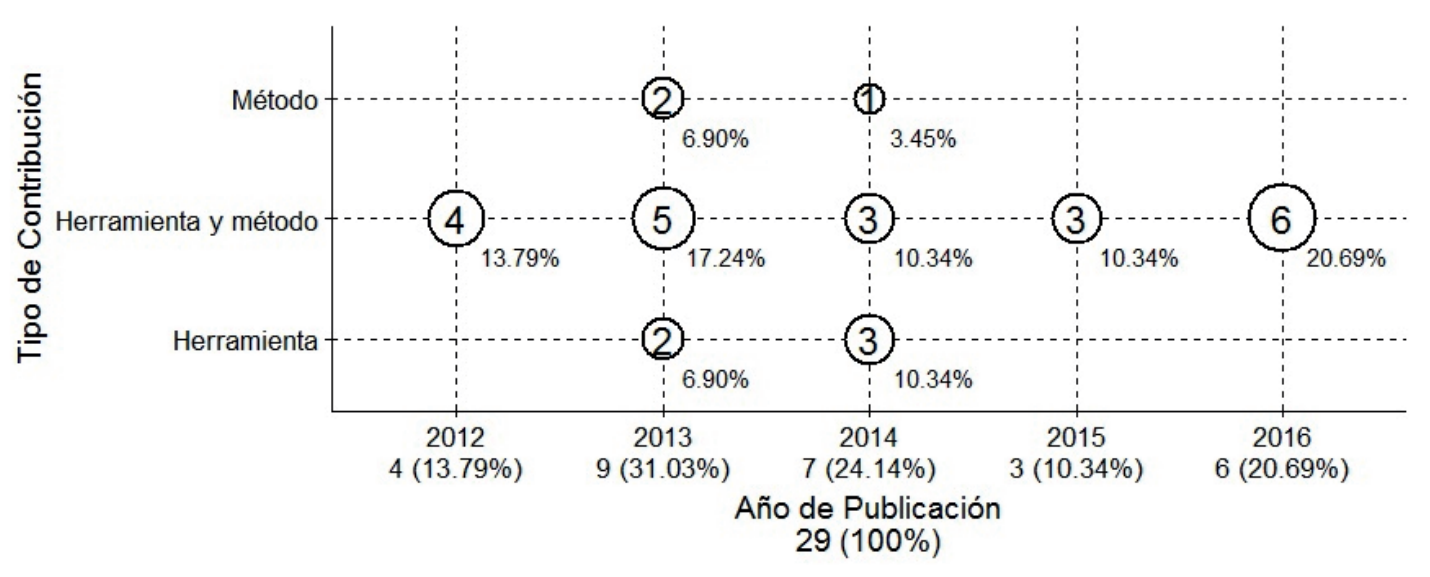

Figura 2: Evolución en el tiempo de las contribuciones.

\section{Análisis y Discusión de Resultados}

RIQ1: ¿Qué tipos de investigación y contribuciones que se han realizado en los últimos 5 años en la arquitectura de software Web Gis Open Source? Como se especifica en la Tabla 4, el 87,10 \% de tipos de investigación en los estudios primarios son Estudios de Casos en los que, en forma exploratoria se estudia proyectos aplicados en la industria. El 9,68 \% realizan experimentos con nuevas componentes o herramientas Open Source.El 3,23\% realizan una Investigación Acción para mejorar algoritmos de procesamiento de información geográfica que se muestran en la Fig. 1. En esta no se incluye 
el 2017 debido a que el mapeo tiene como fecha de corte el mes de junio de 2017, razón por la cual el año no estaría completo, pudiendo distorsionar la estadística de tendencias de investigaciones y contribuciones. Además, en la Fig. 1 se evidencia que en el 2013 se realizaron la mayor cantidad de investigaciones y existe una tendencia incremental de las investigaciones en al ámbito del presente artículo y para finalizar, en su mayoría las investigaciones son Estudios de Casos.

En la Tabla 5 se indica que el $74,19 \%$ de los estudios contribuyen explorando arquitecturas en base a la integración de componentes open source, incluyendo un componente de desarrollo propio en algunos casos y de acuerdo a un método establecido según el área de aplicación del software. El 16,13\% incluye en las arquitecturas estudiadas componentes nuevos y propios para un estudio exploratorio o experimento. El 9,68\% plantea métodos propios para estudiar y mejorar algoritmos.

Se analizó también los principales componentes de las arquitecturas open source explorados en las 31 investigaciones primarias, en los siguientes ámbitos:

- Librerías de Clientes Web.

- Servidores de Mapas.

- Bases de datos.

- Lenguaje de programación Back end.

TABLA 6: Librerías de Clientes Web.

\begin{tabular}{|c|c|c|c|}
\hline Librería & Cantidad & Porcentaje \% & Referencias \\
\hline Open Layers & 9 & 29,03 & $\begin{array}{c}{[1],[3],[8],[14],[16],[18],[21],} \\
{[29],[30]}\end{array}$ \\
\hline $\begin{array}{l}\text { GeoExt y Open } \\
\text { Layers }\end{array}$ & 5 & 16,13 & {$[10],[20],[22],[24],[31]$} \\
\hline Adobe Flex & 2 & 6,45 & {$[5],[6]$} \\
\hline Leaftlet & 2 & 6,45 & {$[26],[27]$} \\
\hline Otros & 8 & 25,81 & $\begin{array}{c}{[4],[9],[12],[15],[19],[23],[25],} \\
{[28]}\end{array}$ \\
\hline No específica & 3 & 9,68 & {$[2],[13],[17]$} \\
\hline No requiere & 2 & 6,45 & {$[7],[11]$} \\
\hline Total & 31 & 100 & \\
\hline
\end{tabular}

En la Tabla 6 se evidencia que las librerías más estudiadas en las arquitecturas de software analizadas, son las librerías de java script Open Layers y en muchos casos, en combinación con las también librerías de java script GeoExt.

En la tabla 7 se muestra que el Servidor de Mapas, GeoServer es el más utilizado para formar parte de arquitecturas que integran componente de software open source, para 
TABla 7: Servidores de Mapas.

\begin{tabular}{l|c|c|c|}
$\begin{array}{l}\text { Servidor de } \\
\text { mapas }\end{array}$ & Cantidad & Porcentaje \% & Referencias \\
Geoserver & 16 & 51,61 & $\begin{array}{l}{[1],[3],[5],[10],[13],[14],[16],[20],} \\
{[21],[23],[24],[25],[26],[27],[30],} \\
{[31]}\end{array}$ \\
\hline $\begin{array}{l}\text { Mapserver } \\
\text { Otros }\end{array}$ & 8 & 25,81 & {$[4],[8],[9],[11],[12],[17],[19],[22]$} \\
No require & 4 & 12,90 & {$[2],[6],[15],[29]$} \\
\hline Total: & 3 & 9,68 & {$[7],[18],[28]$}
\end{tabular}

la renderización de información geográfica y la generación de servicios web geográficos. Mientras, en la tabla 8 se evidencia que el lenguaje Php con un $22.58 \%$ es el lenguaje de Back End más usado en los estudios de arquitecturas revisadas, seguido por el lenguaje Java, con un 12\%. En la tabla 9 se muestra que la base de datos Postgresql, con su extensión espacial Postgis es la principal herramienta de base de datos open source, para el almacenamiento y la gestión de la información geográfica. Para finalizar, existen otros componentes Open Source, que también son estudiados, pero en proyectos orientados a temáticas específicas, como por ejemplo redes geométricas, redes viales, etc.

TABLA 8: Lenguaje de programación Back end.

Lenguaje
Java
Python
Php
Net
Otros
No requiere o no
espeficia
Total

\begin{tabular}{|c|c|}
\hline Cantidad & Porcentaje \% \\
\hline 4 & 12,90 \\
\hline 2 & 6,45 \\
\hline 7 & 22,58 \\
\hline 2 & 6,45 \\
\hline 2 & 6,45 \\
\hline 14 & 45,16 \\
\hline 31 & 100 \\
\hline
\end{tabular}

Referencias
[8], [11], [15], [16]
$[10],[22]$
$[9],[12],[17],[18],[19],[21],[23]$
$[25],[29]$
$[6],[7]$
$[1],[2],[3],[4],[5],[13],[14],[20]$,
$[24],[26],[27],[28],[30],[31]$

TABLA 9: Bases de datos.

\begin{tabular}{l|c|c|}
\hline $\begin{array}{l}\text { Base de datos } \\
\text { Postgresql con } \\
\text { postgis }\end{array}$ & 24 & 77,42 \\
\hline Sql express & 1 & 3,23 \\
\hline No require & 3 & 6,45 \\
\hline No especifica & 2 & 6,45 \\
\hline Otras & 1 & 3,23 \\
\hline Total & 31 & 100
\end{tabular}

\section{Referencias}

[1], [3], [4], [5], [7], [8], [9], [10], [12], [13], [14], [16], [17], [18], [19], $[20],[21],[22],[23],[24],[26],[27]$, [30], [31]

[29]

[2], [11], [28]

[6], [25]

[15] 


\section{Amenazas a la Validez}

Los principales riegos de la validez de la investigación se relacionan con el sesgo de la selección de los estudios; en otros casos a posibles inexactitudes en la extracción de los datos; y, podría ser también a que no hemos podido localizar todos los estudios primarios. Sin embargo, pese a que no es posible llegar a una validez total se ha realizado las siguientes acciones para minimizar posibles inexactitudes:

Se estableció una metodología probada y detallada en la sección "Pregunta de invegación y metodología" para establecer la cadena de búsqueda de los artículos.

Se realizó un proceso de inclusión y exclusión definido y analizado por expertos informáticos y especializados en el área de las tecnologías de los sistemas de información geográficos mediante la estrategia de uso de palabras clave para desarrollar nuestro propio esquema de clasificación, proceso que se realizó leyendo los resúmenes, el contenido de artículo y buscando palabras clave y conceptos que reflejen la contribución del artículo, que ayudó a definir un conjunto de categorías que es representativo del contexto de investigación.

\section{Conclusiones}

Con el EMS presentado en este artículo se ha identificado las principales investigaciones de arquitecturas de software open source para sistemas de información geográficos, publicadas desde el año 2012 hasta el mes de junio de 2017, en las librerías digitales de: IEEE Xplore y Scopus. Este tipo de estudios de mapeo es básico para posteriormente facilitar la apertura del campo a nuevos investigadores, ya que sus resultados pueden ser utilizados para identificar tendencias y brechas en la investigación, poniendo de manifiesto las enormes posibilidades de trabajo para la comunidad de los investigadores en las tecnologías web de los sistemas de información geográficos, en este contexto, la investigación de arquitecturas de software de sistemas informáticos open source, están enfocadas en un $74,19 \%$, en estudios de casos que presentan en forma exploratoria las arquitecturas de software usadas para solventar proyectos GIS con problemáticas diversas y en ámbitos diversos como: Riesgos naturales, emisiones, uso de tierras, etc. El 25,81\% realiza investigaciones para integrar nuevos componentes y nuevos algoritmos que mejoren diferentes aspectos de la arquitectura. De allí, la necesidad de realizar nuevas investigaciones (Experimentos y Investigaciónacción) orientadas a evaluar y mejorar los componentes de la arquitectura de software Open Source de un sistema de información geográfico en un entorno Web. 
Adicional también en el presente artículo (Fig. 1 y Fig 2), se sintetiza la evolución en el tiempo de las investigaciones y contribuciones en el ámbito estudiado, a través de lo cual se evidencia que en el año 2013 se realizaron más investigaciones, pero sin embargo también se evidencia que existe una clara tendencia incremental de la ejecución de estudios científicos en el área del presente artículo.

Por último, el presente estudio contribuye con una sistematización de componentes y herramientas de software usados en las investigaciones de arquitecturas llevadas a cabo durante los últimos 5 años, lo cual, puede resultar de mucha utilidad no solo a investigadores, sino también a profesionales relacionados a las tecnologías de los sistemas de información geográficos.

\section{Referencias}

[1] Frank Carrera Calderón (2017), UNIANDES y la Infraestructura de Datos Espaciales.

[2] IGAC (2008), Fundamentos de Sistemas de Información Geográfica. CIAF.

[3] Anderson, G., \& Moreno-Sanchez, R. (2003). Building web-based spatial information solutions around open specifications and open source software. Transactions in GIS, 7(4), 447-466.

[4] Hecht L 2002 Insist on interoperability. GeoWorld 15(4): 22 - 3

[5] Dangermond, J., 2002. Web services and gis. Geospatial Solutions, 12 (7), 56.

[6] Hecht, L., 2002. Web services are the future of geo-processing. GeoWorld, 15 (6), $23-4$.

[7] Ye, Z. (2009). A Web-Based geographical information system prototype on Portuguese traditional food products (Doctoral dissertation).

[8] Taboada González, José; José, Cotos Yáñez (2005), Sistemas de Información Medio Ambiental. Netbiblo.

[9] Ruíz Lasanta, Maríadel Bueyo (2010), Diseño y desarrollo de un cliente Web Processing Service (WPS) para gvSIG.

[10] Petersen, K., Feldt, R., Mujtaba, S., \& Mattsson, M. (2008, June). Systematic Mapping Studies in Software Engineering. In EASE (Vol. 8, pp. 68-77).

[11] Petersen, K., Feldt, R., Mujtaba, S., \& Mattsson, M. (2008). Systematic mapping studies in software engineering. 12th International Conference on Evaluation and Assessment in Software Engineering, 171.

[12] Petticrew, M., \& Roberts, H. (2008). Systematic reviews in the social sciences: A practical guide Wiley-Blackwell. 
[13] Beecham, S., Baddoo, N., Hall, T., Robinson, H., \& Sharp, H. (2006). Protocol for a systematic literature review of motivation in software engineering. University of Hertfordshire

[14] Ruíz Lasanta, Maríadel Bueyo (2010), Diseño y desarrollo de un cliente Web Processing Service (WPS) para gvSIG.

[15] Taboada González, José; José, Cotos Yáñez (2005), Sistemas de Información Medio Ambiental. Netbiblo.

\section{Referencias de estudios primarios incluidos en el presente estudio}

[1] Mainak Bandyophadyay, Maharana Pratap Singh, Varun Singh (2012). Integrated visualization of distributed spatial databases An open source Web-GIS approach. 1st Int'I Conf. on Recent Advances in Information Technology.

[2] Zeqiang Chen, Nengcheng Chen, Chao Yang, and Liping Di (2012). Cloud Computing Enabled Web Processing Service for Earth Observation Data Processing. IEEE journal of selected topics in applied earth observations and remote sensing, vol. 5, no.

[3] Yu Nakayama, Suguru Mori (2012). FOSS4G based mobile Web-GIS for field survey in natural environmental studies. 9th International Conference on Ubiquitous Intelligence and Computing and gth International Conference on Autonomic and Trusted Computing.

[4] Maria Teresa Françoso, Diogenes Cortijo Costa, Mariana Mello Valin and Rogério Rodrigues Amarante (2013). Free software for development of Web GIS in tourism accessibility. Applied Mechanics and Materials Vols 256-259 (2013) pp 2953-2956.

[5] Guiyun Zhou, Jiayuan Lin, Wenfeng Zheng (2012). A web-based geographical information system for crime mapping and decision support. Computational Problem-Solving (ICCP).

[6] Wu Kehe, Cheng Jiabo, Chen Wei, Zhang Chi (2013). Design and Implementation of Open Source WebGIS Client Framework Based on Flex. International Conference on Computational and Information Sciences.

[7] Jianghua Zheng, Zhangang Zhang, Błażej Cie>płuch, Adam C. Winstanley, Peter Mooney and Ricky Jacob (2013). A PostGIS-based pedestrian way finding module using OpenStreetMap data. Geoinformatics (GEOINFORMATICS), 2013 21st International Conference.

[8] Juan Porta, Jorge Parapar, Paula García, Gracia Fernandez, Juan Tourino, Ramon Doallo, Francisco Onega, Ines Sant, Pablo Díaz, David Miranda, Rafael Crecente 
(2013). Web-GIS tool for the management of rural land markets: Application to the Land Bank of Galicia (NWSpain). Earth Sci Inform.

[9] Dimitrios Gkatzoflias, Giorgos Mellios, Zissis Samaras (2013). Development of a web GIS application for emissions inventory spatial allocation based on open source software tools. Computers \& Geosciences 52 (2013) 21-33.

[10] S. Balbo, P. Boccardo, S. Dalmasso, and P. Pasquali (2013). A public platform for geospatial data sharing for disaster risk management. The International Archives of the Photogrammetry, Remote Sensing and Spatial Information Sciences, Volume $\mathrm{XL}-5 / \mathrm{W}_{3}, 2013$.

[11] B. Bessaa, M. Belhadj Aissa, R. Amara and A. Belhadj Aissa (2013). Spatial indexing of static maps for navigation in online GIS: Application for tourism web GIS. Int. J. Computer Applications in Technology, Vol. 47, Nos. 2/3, 2013.

[12] M. Mangiameli, G. Mussumeci (2013). Real time integration of field data Intoa GIS platform for the management of hydrological emergencies. The International Archives of the Photogrammetry, Remote Sensing and Spatial Information Sciences, Volume XL-5/W3, 2013.

[13] S. Cinnirella, F. D'Amore, P. Mazzetti, S. Nativi and N. Pirrone (2013). A spatial data infrastructure for the global mercury observation system. E3S Web of Conferences 28001

[14] Moshiur Moshi, Nadia Nahar†, Rayhanur Rahman and Kazi Sakib (2014). MapBeing: An architecture for manipulating and publishing vector data in web based Geographic Information System. Software, Knowledge, Information Management and Applications (SKIMA), 2014 8th International Conference

[15] Olmo Zavala-Romero, Arsalan Ahmed, Eric P. Chassignet, Jorge Zavala-Hidalgo, Agustin Fernandez Eguiarte, Anke Meyer-Baese (2014). An open source Java web application to build self-contained web GIS sites. Environmental Modelling \& Software 62 (2014) $210 e 220$.

[16] Blagoj Delipetrev,Andreja Jonoski, Dimitri P. Solomatine (2014). Development of a web application for water resources based on open source software. Computers \& Geosciences 62 (2014) 35-42.

[17] Mingyi Duan, Yajun Yang, Haibo Yang (2014). Educational geographic information system based on WebGIS. 2013 International Conference on Information Science and Cloud Computing Companion.

[18] Nik Mohd Ramli Nik Yusoff, Helmi Zulhaidi Mohd Shafri, Ratnasamy Muniandy and Islam Mohammad Wali (2014). Development of obstacle avoidance technique in 
web-based geographic information system for traffic management using open source software. Journal of Computer Science

[19] DOU Changyong, GUO Huadong, HAN Chunming, and LIU Ming (2014). An open source software and web-GIS based platform for airborne SAR remote sensing data management, distribution and sharing. 35th International Symposium on Remote Sensing of Environment (ISRSE35).

[20] Zar Chi Aye, Michel Jaboyedoff, Marc-Henri Derron and Cees J. van Westen (2015). Prototype of a web-based participative decision support platform in natural hazards and risk management. ISPRS Int. J. Geo-Inf. 2015.

[21] Gang Han, Jun Chen, Chaoying He, Songnian Li, Hao Wu, Anping Liao, Shu Peng (2014). A web-based system for supporting global land cover data production. ISPRS Journal of Photogrammetry and Remote Sensing.

[22] Achim Knörchen n, Gunnar Ketzler, Christoph Schneider (2015). Implementation of a near-real time cross-border web-mapping platform on airborne particulate matter (PM) concentration with open-source software. Computers \& Geosciences 74 (2015) 13-26.

[23] Rabindra K. Barik, Pratyush K. Das, Rakesh K. Lenka (2016). Development and implementation of SOA based SDI model for tourism information infrastructure management web services. Cloud System and Big Data Engineering (Confluence), 2016 6th International Conference.

[24] Nizamuddin, Hizir, Ardiansyah, D Pertiwi and P Handayani (2016). Development of Web GIS for information of renewable energy in Aceh Province after rehabilitation and reconstruction process. IOP Conference Series: Earth and Environmental Science, Volume 56, conference 1.

[25] Pratap Sakhare, Sweedle Mascarnes, Anita Chaudhari (2015). Development of WebGIS framework for Indian technical institutes using open source GIS tools. Computer, Communication and Control (IC4), 2015 International Conference on.

[26] M. A. Brovelli, F. C. Fahl, M. Minghini, M. E. Molinari (2016). Land user and land cover maps of Europe: A webgis platform. The International Archives of the Photogrammetry, Remote Sensing and Spatial Information Sciences, Volume XLIB7, 2016.

[27] Matteo MATTAVELLI, Daniele STRIGARO, Ivan FRIGERIO, Filippo LOCCl, Maria Teresa MELIS \& Mattia DE AMICIS (2016). The IDB: An ice core geodatabase for paleoclimatic and glaciological analyses. Revista GEOGRAFIA FISICA E DINAMICA QUATERNARIA artículo 1. 
[28] Nurin Swasti Kanthi and Taufik Hery Purwanto (2016). Application of OpenStreetMap (OSM) to Support the Mapping Village in Indonesia. IOP Conference Series: Earth and Environmental Science, Volume 47, Number 1.

[29] Dekui Lv, Xiaxin Ying, Xibao Gao, Wei dong Tao, Yanjun Cui, Tingting Hua (2016). A WebGIS platform design and implementation based on Open Source GIS middleware. Geoinformatics, 2016 24th International Conference on

[30] Solange Duruz , Christine Flury, Giona Matasci, Florent Joerin, Ivo Widmer, Stéphane Joost (2017). A WebGIS platform for the monitoring of Farm Animal Genetic Resources (GENMON).

[31] Nizamuddin, Hizir, Ardiansyah, D Pertiwi and P Handayani (2017). Development of Web GIS for information of renewable energy in Aceh Province after rehabilitation and reconstruction process. IOP Conference Series: Earth and Environmental Science, Volume 56, conference 1. 